\title{
Motions capture system to assess intraoperative staff movements and door openings: \\ Impact on surrogates of the infectious risk in surgery.
}

\section{Authors' names}

Gabriel Birgand, $\mathrm{PhD}^{1,2,3}$; Christine Azevedo, $\mathrm{PhD}^{4,5}$; Stephane Rukly, MSc ${ }^{1}$; Roger PissardGibollet, $\mathrm{PhD}^{5}$; Gaëlle Toupet, MSc ${ }^{3}$, Jean-François Timsit, MD ${ }^{1,2,6}$, Jean-Christophe Lucet, MD $^{1,2,3}$ and the ARIBO Study Group ${ }^{7}$

\section{Authors' affiliations}

${ }^{1}$ INSERM, IAME, UMR 1137, F-75018 Paris, France

${ }^{2}$ Univ Paris Diderot, IAME, UMR 1137, Sorbonne Paris Cité, F-75018 Paris, France

${ }^{3}$ AP-HP, Hôpital Bichat, Infection Control Unit, F-75018 Paris, France

${ }^{4}$ INRIA / Laboratoire d'Informatique de Robotique et de Microélectronique de Montpellier (LIRMM), 860 Rue Saint Priest - 34095 Montpellier cedex 5, France

${ }^{5}$ Institut National de Recherche en Informatique et en Automatique, Montbonnot, France;

${ }^{6}$ AP-HP, Hôpital Bichat, Medical Intensive Care Unit, F-75018 Paris, France

${ }^{7}$ Members of the ARIBO study group:

The following contributors provided and cared for study patients: Pierre SQUARA, Corinne DE DIESBACH, Alain BRUSSET, Marie-Françoise VOGEL, François GOUIN, Sophie TOUCHAIS, Jacqueline LEPENNEC, Gérard BABATASI, Emmanuel DE THOMASSON, Mathieu DEBAUCHEZ, Christian MAZEL, Pascal BIZOT, Philippe ROSSET, Patrick NATAF, Philippe MASSIN, Agnès JUE-DENIS

The following contributors collected data: Gilles ANTONIOTTI, Philippe SOUCHOIX, Xavier RICHOMME, Marie-Noëlle DESCHAMPS, Didier LEPELLETIER, Florence LEGALLOU, 
Nathalie FERRONNIERE, Audrey MOUET, Xavier LECOUTOUR, Véronique AGUELON, Claire LESTEVEN, Carole PORNET, Jean Baptiste STERN, Jacques-Yves NIZOU, Yves-Marie VANDAMME, Maurice TANGUY, Marie-Laure JOLY-GUILLOU, Nathalie VAN DER MEE MARQUET, Aurélie THOMAS-HERVIEUX

Correspondence to Gabriel Birgand; gbirgand@gmail.com

Infection control Unit, Bichat-Claude Bernard University Hospital, Assistance PubliqueHôpitaux de Paris, and Paris 7 Denis Diderot University, 46 rue Henri Huchard 75018 Paris,

Meeting presentation: European Congress of Clinical Microbiology and Infectious Diseases, Copenhagen 2016.

Brief title: Surgical staff dynamics and infectious risk

Word count: 3020 words 


\begin{abstract}
Objectives: We longitudinally observed and assessed the impact of the operating room (OR) staff movements and door openings on surrogates of the exogenous infectious risk using a new technology system.
\end{abstract}

Design and setting: This multicentre observational study included 13 ORs from 10 hospitals, performing planned cardiac and orthopaedic surgery (total hip or knee replacement). Door openings during the surgical procedure were determined from data collected by inertial sensors fixed on the doors. Intraoperative staff movements were captured by a network of eight infra-red cameras. For each surgical procedure, 3 microbiological air counts, longitudinal particles counts, and one bacteriological sample of the wound before skin closure were performed. Statistics were performed using linear mixed model for longitudinal data.

Results: We included 34 orthopaedic and 25 cardiac procedures. The median frequency of door openings from incision to closure was independently associated with an increased $\log _{10} 0.3 \mu \mathrm{m}$ particle (ß: 0.03, SD: 0.01, $\mathrm{p}=0.01)$ and air microbial count ( $($ : $0.07, \mathrm{SD}: 0.03, \mathrm{p}=0.03)$, but not significantly correlated with the wound contamination before closure $(r=0.13, p=0.32)$. The number of persons ( $(3:-0.08, \mathrm{SD}: 0.03, \mathrm{p}<0.01)$, and the cumulated movements by the surgical team (ß: $0.0004, \mathrm{SD}: 0.0005, \mathrm{p}<0.01$ ) were associated with $\log _{10} 0.3 \mu \mathrm{m}$ particle counts.

Conclusions: This study demonstrated a previously-missing association between intraoperative staff movements and surrogates of the exogenous SSI risk. Restriction of staff movements and door openings should be considered for the control of the intraoperative exogenous infectious risk. 


\section{INTRODUCTION}

Surgical site infections (SSI) are the most common hospital-acquired infections among surgical patients, the leading cause of hospital readmission after surgery with a major financial burden. ${ }^{1,2}$ It is generally accepted that the contamination of the surgical wound mainly occurs at the time of surgical procedure in the operating room (OR), eventually leading to SSI. Main routes of microbial entry into an open clean surgical wound include from the patient's skin or the surgical staff, by airborne microbes and by contaminated surgical instruments. ${ }^{3}$

The literature suggests an impact of surgical team behavior on the air microbial contamination and the SSI risk. ${ }^{4}$ Door openings have been demonstrated to adversely affect air exchange, air quality, and positive pressure in the OR, affecting the air microbial contamination in the OR. ${ }^{5}$ Current guidelines do not include specific recommendations regarding the best $\mathrm{OR}$ staff behavior (except for clothing rules and hand hygiene) to decrease the exogenous risk of SSI. ${ }^{6,7}$ New technologies using motion capture systems present an opportunity to objectively and continuously assess the global OR staff dynamics and behavior during surgical intervention in $\mathrm{OR}^{8}$

This study aimed to objectively describe and assess staff behaviors in the OR and their variability by recording staff movements using a motion tracking system and door openings detection system, and to assess correlations between movements of the OR personnel and the SSI risk, as approximated by surrogates of the exogenous infectious risk, in a panel of ORs from two clean surgical specialties. 


\section{METHODS}

\section{Population and location of the study}

This observational multicenter study was carried out at 10 facilities (five university hospitals, five private hospitals) located in France, and a convenience sample of 13 ORs, six in cardiac surgery and seven in orthopedic surgery. ${ }^{9}$ Procedures requiring full median sternotomy and total hip (THR) and knee replacement (TKR) were included. The population observed was formed of OR personals and any other person likely to enter the OR during surgical procedure. At the preoperative stage, patients were informed orally by surgeons, anesthetists or infection control specialists of the ongoing study and an information letter was systematically given. A waiver of informed consent was granted for patient. However, consents were obtained from the OR members. The study was approved by an ethics committee.

\section{System of motion capture}

A technology of motion capture based on a video tracking system was adapted for the objective, continued and prolonged detection and characterization of movements in the OR. A network of eight video cameras (VICON-Bonita ${ }^{\circledR}$, Vicon - Los Angeles, USA) ${ }^{10}$ was fixed upright to the wall using suction cup supports. Markers placed on the surgical caps/hoods of each person entering the OR were located in 3D using the Vicon Tracker software ${ }^{\circledR}$ by a method of spatial triangulation. ${ }^{11} 68$ LEDs situated on each camera produced an infrared light reflected by hemispherical markers and acquired by the optic. The detection of the same marker by different 
cameras allows its three-dimensional (3D) positioning. The motion capture was performed by a continuous tracking of reflective markers placed on the surgical caps/hoods of each person entering the OR.

Different markers distinguished different professional categories: surgeons, anesthesiologists (doctors, nurse and extracorporeal circulation personal), OR nurses and others (including visitors). A study coordinator holding a marker stayed in the OR during the procedures moving only for the sampling and to provide technical assistance.

Two autonomous wireless inertial sensors (HiKoB FOX ${ }^{\circledR}$, HiKoB - Villeurbanne, France) were fixed on each door of the OR and synchronized with the motion tracking system. Door openings were determined offline based on data collected by the inertial sensors.

The motion tracking system stayed during one week in the same OR to get people used to it and to take into account potential behavioral modifications due to the Hawthorne effect. Data acquisition started at skin incision and continued until wound closure. Door openings sensors were kept in a sample of OR for one additional week after removal of video camera. OR staff was not informed of the persistence of doors sensors. Thus, the comparison of the frequency of door openings during and after removal of the motion tracking system allowed estimating the impact of the Hawthorne effect.

\section{Surrogates of the infectious risk}

Microbiological air counts were measured using an impactor air sampler (Air-test Omega, LCB, La Salle France) at a flow rate of $100 \mathrm{~L} / \mathrm{min}$ for 5 min $(500 \mathrm{~L})$ on trypticase soy agar (BioMerieux, France), which was then incubated for four days at $30^{\circ} \mathrm{C}$. Air counts were 
expressed as colony-forming units $(\mathrm{CFU})$ per $\mathrm{m}^{3}$. Samples were taken at the time of skin incision, 15 min after bone cut (sternum or femur) and at wound closure.

Particle count (HandiLaz ${ }^{\circledR}$ Mini, Boulder, CO, USA) was performed using a

photodetection device continuously from incision to wound closure. ${ }^{12}$ The particle analyzer sampled for $1 \mathrm{~min}$ every $5 \mathrm{~min}$ from the patient entry to exit from the OR at a rate of $28.3 \mathrm{~L} / \mathrm{min}$ $\left(1.0 \mathrm{ft}^{3} / \mathrm{min}\right)$. Particles were classified by diameter into 3 sizes: $0.3 \mu \mathrm{m}, 0.5 \mu \mathrm{m}$ and $5 \mu \mathrm{m}$. Both particle and microbiological air counts were performed at the patient's head.

A sample from the operated wound was performed before closure and prior to antiseptic aspersion. We used the sampling method previously described ${ }^{13}$ using sterile pads of polyamidepolyester-viscose placed on subcutaneous tissue for $1 \mathrm{~min}$. Microorganisms were extracted by vortexing the pads in phosphate buffer (PBS) with Tween 80 at 2\% and lecithin at $0.3 \%$ (Hyphen BioMed, Neuville sur Oise, France). For each pad, an aliquot of $0.5 \mathrm{ml}$ of phosphate buffer was cultured on blood agar after $48 \mathrm{~h}$ of aerobic and anaerobic incubation, and colonies were counted without further identification.

\section{Data collection}

Information was collected on: (i) the surgical procedure, including the surgical specialty, procedure and technique used, incision time, preselected procedure periods described above and closure time; (ii) surgical environment characteristics, including type of air filtration, either laminar airflow (LAF) or turbulent, air changes per h, positive pressure and the class of air cleanliness for airborne particulate level (ISO 14644). The architecture of the OR was also collected, including size and volume. 


\section{Statistical analysis}

The results of particle counts were $\log _{10}$ transformed. Numbers of CFU cultured from wounds in aerobic and anaerobic media were added up and computed to obtain the number of CFU per square centimeter of wounds. Results of the wound culture were categorized into 3 classes: negative culture, $1-10$, and $>10 \mathrm{CFU} / 100 \mathrm{~cm}^{2}$. Microbiologic air counts were also categorized into the following 3 different classes: negative, $1-10$, and $>10 \mathrm{CFU} / \mathrm{m}^{3}$. These stratifications were performed using the $25^{\text {th }}$ and the 75 th percentiles distributions. Continuous variables were compared using Mann-Whitney and proportion using Chi-square tests, as appropriate.

To determine potential risk factors for an increase of particles and air microbial counts, univariate linear mixed models for longitudinal data with a random intercept for each intervention and each OR and a random slope for time were used. The covariance structure « unstructured» were used for the random effects. The Satterthwaite method was used to compute the denominator degrees of freedom for the tests of fixed effects. Behaviors observed (numbers of door openings, number of persons, the total movements by persons) during the five minutes period before the particle count (corresponding to the period between two particle counts) was considered to estimate associations. This time period was pragmatically selected to consider the

quasi-instantaneous ${ }^{26}$ impact of door openings on the positive pressure and airflow in the OR, and to leave enough time to get explicative events. (e.g. door openings) 
Significant variables at 0.1 were selected for the multivariate model. A backward selection was used on the multivariate model. Conditional studentized residuals were checked. A sub analysis was performed on interventions with video data to precisely evaluate the effect of number of persons and staff movements on increase of particles. ${ }^{14}$

The same method was applied to determine potential risk factors for an increase of air microbial count. Unlike the previous model, only 3 measures of air microbial count were done. Behaviors observed between the patient's arrival and the first measure, the first and the second measure and the second and the third measure were considered to estimate associations.

(Appendix Figure A1) SAS version 9.3 statistical software (SAS Institute, Cary, NC) was used to perform all analyses.

\section{RESULTS}

\section{General data}

A total of 62 surgical procedures were observed from the $14^{\text {th }}$ May to the $20^{\text {th }}$ December 2013. Three procedures were excluded due to an incomplete data collection, leading to the inclusion of 59 procedures (25 in cardiac and 34 in orthopedic surgery) for the door openings assessment. Data on intraoperative staff movements were comprehensively collected during 34 of the 59 procedures (Figure 1).

The architecture of the 13 participating ORs was characterized by a median surface of 42 (IQR, 36-47) $\mathrm{m}^{2}$, including a median of 2 doors (range 1 to 5 ). The air ventilation system was 
turbulent in $8 / 13$ ORs (6/7 in cardiac surgery and 2/6 in orthopedic surgery). The median baseline air renewal was 53 (45-64) changes per hour, with a median positive pressure of 19 (12-33) Pa.

In cardiac surgery, only the first procedure of the day in the OR was included, whereas in orthopedic surgery 19 procedures were in $1^{\text {st }}$ position, 11 in $2^{\text {nd }}$ and 4 in $3^{\text {rd }}$ position during the same day. In orthopedic surgery, the median duration (IQR) from patient entry to exit and from incision to closure was $2.5 \mathrm{~h}(2-3.1)$ and $1.3 \mathrm{~h}(1-1.8)$, respectively. In cardiac surgery, the same figures were $5.1 \mathrm{~h}(4.7-6.2)$ and $3.5 \mathrm{~h}(3-4.3)$, respectively (Table 1).

\section{Surrogates of the infectious risk}

The median $\log _{10}$ of $0.3 \mu \mathrm{m}, 0.5 \mu \mathrm{m}$ and $5 \mu \mathrm{m}$ of the 1747 particle counts performed measured during the 59 procedures are displayed in supplementary Table S1 and Figure 2A. The counts of $0.3 \mu \mathrm{m}$ particles varied according to ORs and procedures. The $\log _{10}$ of $0.3 \mu \mathrm{m}$ varied according to ORs and procedures, with a mean in ORs with LAF of $6.8(\mathrm{SD}, 1)$ and $6.8(\mathrm{SD}, 0.9)$ during orthopedic procedures. These values were consistently below those observed in ORs with turbulent ventilation systems (mean, 7.2; SD, 0.9) and during cardiac surgery (mean, 7.3; SD, 0.9) $(\mathrm{p}<0.01)$ (Appendix Figure A2).

The median (IQR) air microbial count at three moments in all 59 procedures was $3(0-8)$ CFUs per $\mathrm{m}^{3}$. Among the 177 air samples, 50 (28\%) were sterile, 90 (51\%) carried 1 to $10 \mathrm{CFUs}$ / $\mathrm{m}^{3}$ and $37(21 \%)>10 \mathrm{CFUs} / \mathrm{m}^{3}$. For this last category, the median number of CFUs in air sampling was $21(14-29$, range $11-47)$ per $\mathrm{m}^{3}, 33 / 37$ in cardiac surgery and $35 / 37$ in OR with a turbulent ventilation system.

Among the 59 cultures of wound samples, 33 (56\% of patients) were sterile, $18(30 \%)$ had 1 to $10 \mathrm{CFUs} / 100 \mathrm{~cm}^{2}$ and $8(14 \%)$ had $>10 \mathrm{CFUs} / 100 \mathrm{~cm}^{2}$. Wounds in orthopedic surgery were 
significantly less contaminated at closure than in cardiac surgery ( 24 vs 9 sterile cultures; 9 vs 11 with 1 to $10 \mathrm{CFUs} / 100 \mathrm{~cm}^{2}$ and 0 vs 6 with $>10 \mathrm{CFUs} / 100 \mathrm{~cm}^{2}$, respectively) $(\mathrm{p}=0.002)$.

\section{Door openings}

Among the 59 procedures observed, the median (IQR) frequency of 19.4 (13.9-25.5) openings per hour, with large variation across ORs (Table 1 and Figure 2B). Doors of aseptic preparation rooms were the most frequently opened and door openings were mainly generated by the anesthetics team and persons not directly involved in the procedure (i.e. assistant nurse or visitors).

During the 34 orthopedic procedures, the median (IQR) frequency was $14.8(12.2-21.2)$ openings per hour from incision to skin closure. Doors stayed opened a cumulated duration of 4.2 (2.6-10.8) minutes, corresponding to $6 \%(3.1-10.4)$ of the incision-to-closure period. During the 25 cardiac procedures, the median frequency of openings was 23.4 (19.7-30) per hour from incision to closure. The cumulated duration of openings was 13.1 (10.7-21.3) minutes, corresponding to $7.3 \%(5.3-10.6)$ of the operating time.

The median frequency of door openings observed after the removal of the video tracking system was $36.6(33.3-42.6)$ per hour from patient entry to exit vs $34.5(23.6-48.8)$ per hour in presence of the cameras in the $\mathrm{OR}(\mathrm{p}=0.5)$ (Appendix Table A2).

\section{Number of persons and staff movements}


Among the 34 procedures (19 orthopedic and 15 cardiac) with the recording of intraoperative staff movements, the median (IQR) number of persons present from incision to skin closure was 10 (8-11) (Table 1). The median (IQR) cumulated time spent by individuals in the OR during a single procedure was $1.7 \mathrm{~h}(1.1-2.4)$. The Figure $2 \mathrm{C}$ displays the disparities of movements by specialty and OR. The cumulated movements by the entire team from incision to skin closure for one surgical procedure represented 12.1 (11.5-14) kilometers. Each member of the team walked in median 373 (324-461) meters from incision to skin closure in orthopedic surgery and $832(629$ - 877) meters in cardiac surgery.

\section{Impact of behaviors on the surrogates of the exogenous infectious risk}

The multivariate linear model performed on door openings collected during the 59 procedures, found a significant positive link between the $\log _{10} 0.3 \mu \mathrm{m}$ particle counts and the number of door openings per period of 5 minutes ( $(3: 0.03, \mathrm{SD}: 0.01, \mathrm{p}=0.01)$. In other words, one door opening during the 5 minutes preceding the particle sampling raised the $\log _{10} 0.3 \mu \mathrm{m}$ particles by 0.03 .

The turbulent airflow and the number of door openings per period were associated with an increased air microbial count ( $(: 8.57, \mathrm{SD}: 3.74, \mathrm{p}=0.04 ; ß: 0.07, \mathrm{SD}: 0.03, \mathrm{p}=0.03$; respectively) (Table 2).

The frequency of door openings and the mean of air bacterial counts from the incision to skin closure period was positively but not significantly correlated with the wound contamination before closure $(\mathrm{r}=0.13, \mathrm{p}=0.32$ and $\mathrm{r}=0.15, \mathrm{p}=0.22$, respectively). 
The multivariate analysis performed on the 34 procedures with data on staff movements showed a significant association between the cumulated movements by the surgical team (ß: 0.003, SD: $0.0004, \mathrm{p}<0.01)$ and the $\log _{10} 0.3 \mu \mathrm{m}$ particle counts (Table 3 Model 1). A subanalysis was performed to assess the relationship between the number of persons and their cumulated movements on the $\log _{10} 0.3 \mu \mathrm{m}$ particle counts. The inverse correlation found between both variables informs of the greater impact of staff movements on the $\log _{10} 0.3 \mu \mathrm{m}$ particle counts compared to the number of persons (Table 3 Model 2).

The univariate analysis of $\log _{10} 0.5$ and $5 \mu \mathrm{m}$ particle counts were significantly associated with the cumulated movements by team during the 5-minute period (ß: 0.003 , SD: $0.0004, \mathrm{p}<0.001 ; \beta: 0.003, \mathrm{SD}: 0.0005, \mathrm{p}<0.001$; respectively), but not with the number of persons. The non-validation of statistical assumptions (residuals not normally distributed) did not allowed to interpret the multivariate analysis. 


\section{DISCUSSION}

Door openings and staff movements appeared highly heterogeneous varying approximately 4-fold according to ORs and procedures in each specialty. Both had a significant impact on the air contamination by particles and microorganisms during procedures. The cumulated movements by the surgical team significantly affected the $\log _{10} 0.3,0.5$ and $5 \mu \mathrm{m}$ particle counts. This association was confirmed in the multivariate analysis for $\log _{10} 0.3 \mu \mathrm{m}$ particle counts. The results of the multivariate model for $\log _{10} 0.5$ and $5 \mu \mathrm{m}$ particle counts were not interpretable due to the non-normal distribution of the residuals.

The variability of behaviors observed despite comparable procedures, may be explained either by the case-mix, a lapse in the discipline of individuals or teams, or by the OR architecture and organization. In the present study, doors were mainly opened by nurses and visitors during orthopedic surgery. In cardiac surgery, anesthetists and external participants contributed to most of door openings. In the literature, most of entries/exits occurring during procedures are explained by the frequent need of supplies or social activities. However, a substantial part remains not explained, suggesting room for improvement. ${ }^{4}$

The results confirm the findings of previous studies suggesting that door openings may affect the air sterility of the OR. ${ }^{15-20}$ Door movements are known to alter the efficacy of ventilation systems by a disruption of the positive pressures ${ }^{5}$, and the air flow. ${ }^{21}$ Our data suggest that controlling the movements of staff members inside the OR may be more efficient than restricting their number to prevent the air particles contamination. (Table 3 Model 2) The

number of airborne particles produced per person has been estimated at $10^{4}$ per minute at rest and 
up to $3.10^{7}$ during exertion. ${ }^{22}$ Thus, a high number of static persons in the OR will consistently generate less airborne particles and bacteria than a restricted number of persons with unregulated movements.

The quantity of microorganisms cultured from the wound before closure was influenced by the cumulated movements by the team but not by the number of door openings. These results have to be taken with caution. A large number of surgical wounds ( $89 \%$ in cardiac surgery) are contaminated at closure. ${ }^{23,24}$ The combination of endogenous and exogenous organisms can confound the relationship between the quantitative presence of organisms in the air and those colonizing the wound during surgery. In addition, the rather low number of wound samplings might not suffice for attaining a statistical association.

A recent meta-analysis concluded that LAF may not be efficient in reducing the risk of SSIs in total hip and knee arthroplasties, and abdominal surgery. ${ }^{25}$ After adjustment, our results showed a significant and independent increase air microbial contamination in OR with conventional airflow system in comparison to LAF. Moreover, the airborne particle concentration was consistently lower at incision in ORs with LAF vs conventional airflow and decreased faster during the procedures (Appendix Figure A2). These findings are feeding the current low quality evidence on the advantage of LAF to prevent SSI in clean surgery.

Our study has several strengths. This is the first multicenter study using motion tracking systems to precisely and continuously assess the intraoperative staff behaviors, including movements inside ORs found to be critical in the present study. The absence of a Hawthorne effect due to the presence of video camera, as compared to a period with door openings collection 
(hidden to staff) but without video cameras suggests the reliability of our results. The cutaneous incision of a sterile site in cardiac and orthopedic surgeries gave the advantage to increase the potential impact of an environmental contamination on the occurrence of subsequent SSI. The high reproducibility of procedures and technique improves the generalizability of the results. Finally, the statistical method allowed adjusting the analysis with a random intercept for each intervention and each operating room and a random slope for time, preventing bias due to important confounding factors.

We acknowledge several limitations. First, the endpoints were surrogates of the environmental infectious risk and not SSI. SSI rate would have been an ideal but unreachable endpoint. Indeed obtaining a benchmarked SSI rate in these surgical units would have required a long duration of surveillance, and many confounding factors should have been collected. Second, air samples were not strictly performed in the sterile site. This bias was minimized by positioning the counters at the patient's head, under the laminar air flow when present, and at a height above sterile drapes separating the sterile site and the anesthesia area. The impact of door openings on the positive pressure and airflow in the OR is quasi-instantaneous. ${ }^{26}$ The five minutes period chosen to analyze the impact of behaviors on the air particle contamination appeared as the best compromise between a period enough large to get events (e.g. door openings) and their closeness to the counts. The longer periods used for the air microbial contamination may more relate to the long term effect of intraoperative behaviors. Longitudinal modelling focused on the $\log _{10} 0.3 \mu \mathrm{m}$ particle and the air microbial counts. A previous study suggested that the 3 types of particle size were strongly correlated with airborne bacterial counts and likely represents a surrogate of overall air contamination during the surgical procedure. ${ }^{27}$ The variability and large values obtained for $0.3 \mu \mathrm{m}$ particles offered the possibility to satisfy the statistical assumptions and precisely model 
and assess the relationship between the traffic flow and the environmental contamination. Finally, $42 \%$ of surgical procedures were excluded from the analysis of staff movement due to noncomprehensive collection of staff positions by the motion tracking system. Moreover, due to the typical duration of cardiac procedures and the amount of time required for study-specific set-up, we only included the first scheduled cardiac procedure, which is potentially not representative of full-day behaviors.

This study highlights the importance of the intraoperative discipline of staff, suggesting that a restriction of staff movements and door openings may prevent the airborne contamination and the associated SSI risk. The awareness of surgical staff in this field may improve behaviors and quality of cares.

Acknowledgements: We thank the bacteriology laboratories that performed bacterial cultures and all the people who participated in the study: We thank Sebastien Bailly for his help in reviewing the statistical method. No preregistration exists for the reported studies reported in this article.

Competing interests: None

Funding: This study was partly supported by the French Ministry of Health (national grant PREQHOS 2011).

\section{Authorship and manuscript preparation}




\section{REFERENCES}

1. Le Manach Y, Collins G, Bhandari M, et al. Outcomes After Hip Fracture Surgery Compared With Elective Total Hip Replacement. JAMA. 2015;314(11):1159-1166.

2. Zimlichman E, Henderson D, Tamir O, et al. Health care-associated infections: a metaanalysis of costs and financial impact on the US health care system. JAMA Intern Med. 2013;173(22):2039-2046.

3. Tammelin A, Hambraeus A, Ståhle E. Routes and sources of Staphylococcus aureus transmitted to the surgical wound during cardiothoracic surgery: possibility of preventing wound contamination by use of special scrub suits. Infect Control Hosp Epidemiol Off J Soc Hosp Epidemiol Am. 2001;22(6):338-346.

4. Birgand G, Saliou P, Lucet J-C. Influence of staff behavior on infectious risk in operating rooms: what is the evidence? Infect Control Hosp Epidemiol. 2015;36(1):93-106.

5. Mears SC, Blanding R, Belkoff SM. Door Opening Affects Operating Room Pressure During Joint Arthroplasty. Orthopedics. 2015;38(11):e991-994.

6. Allegranzi B, Zayed B, Bischoff P, et al. New WHO recommendations on intraoperative and postoperative measures for surgical site infection prevention: an evidence-based global perspective. Lancet Infect Dis. 2016;16(12):e288-e303.

7. Berríos-Torres SI, Umscheid CA, Bratzler DW, et al. Centers for Disease Control and Prevention Guideline for the Prevention of Surgical Site Infection, 2017. JAMA Surg. May 2017.

8. Lucet J-C, Laouenan C, Chelius G, et al. Electronic sensors for assessing interactions between healthcare workers and patients under airborne precautions. PloS One. 2012;7(5):e37893.

9. Birgand G, Azevedo C, Toupet G, et al. Attitudes, risk of infection and behaviours in the operating room (the ARIBO Project): a prospective, cross-sectional study. BMJ Open. 2014;4(1):e004274.

10. Isableu B, Hansen C, Rezzoug N, Gorce P, Pagano CC. Velocity-dependent changes of rotational axes during the control of unconstrained 3D arm motions depend on initial instruction on limb position. Hum Mov Sci. 2013;32(2):290-300.

11. Birgand G, Azevedo $\mathrm{C}$, Toupet $\mathrm{G}$, et al. Attitudes, risk of infection and behaviours in the operating room (the ARIBO Project): a prospective, cross-sectional study. BMJ Open. 2014;4(1):e004274.

12. Dharan S, Pittet D. Environmental controls in operating theatres. J Hosp Infect. 2002;51(2):79-84. 
13. Hambraeus A, Hoborn J, Whyte W. Skin sampling-validation of a pad method and comparison with commonly used methods. J Hosp Infect. 1990;16(1):19-27.

14. Littell, Milliken RC GA, Stroup WW, Wolfinger RD, Schabenberger O. SAS for mixed models. In: 2nd edition. Cary, NC, USA; 2006.

15. Andersson AE, Bergh I, Karlsson J, Eriksson BI, Nilsson K. Traffic flow in the operating room: an explorative and descriptive study on air quality during orthopedic trauma implant surgery. Am J Infect Control. 2012;40(8):750-755.

16. Tjade $\mathrm{OH}$, Gabor I. Evaluation of airborne operating room bacteria with a Biap slit sampler. J Hyg (Lond). 1980;84(1):37-40.

17. Scaltriti S, Cencetti S, Rovesti S, Marchesi I, Bargellini A, Borella P. Risk factors for particulate and microbial contamination of air in operating theatres. J Hosp Infect. 2007;66(4):320-326.

18. Agodi A, Auxilia F, Barchitta M, et al. Operating theatre ventilation systems and microbial air contamination in total joint replacement surgery: results of the GISIO-ISChIA study. $J$ Hosp Infect. 2015;90(3):213-219.

19. Erichsen Andersson A, Petzold M, Bergh I, Karlsson J, Eriksson BI, Nilsson K. Comparison between mixed and laminar airflow systems in operating rooms and the influence of human factors: Experiences from a Swedish orthopedic center. Am J Infect Control. 2014;42(6):665-669.

20. Mathijssen NMC, Hannink G, Sturm PDJ, et al. The Effect of Door Openings on Numbers of Colony Forming Units in the Operating Room during Hip Revision Surgery. Surg Infect. 2016;17(5):535-540.

21. Brohus H, Balling KD, Jeppesen D. Influence of movements on contaminant transport in an operating room. Indoor Air. 2006;16(5):356-372.

22. Hambraeus A. Aerobiology in the operating room--a review. J Hosp Infect. 1988;11 Suppl A:68-76.

23. Bernard L, Sadowski C, Monin D, et al. The value of bacterial culture during clean orthopedic surgery: a prospective study of 1,036 patients. Infect Control Hosp Epidemiol Off J Soc Hosp Epidemiol Am. 2004;25(6):512-514.

24. Tammelin A, Hambraeus A, Ståhle E. Source and route of methicillin-resistant Staphylococcus epidermidis transmitted to the surgical wound during cardio-thoracic surgery. Possibility of preventing wound contamination by use of special scrub suits. $J$ Hosp Infect. 2001;47(4):266-276.

25. Bischoff P, Kubilay NZ, Allegranzi B, Egger M, Gastmeier P. Effect of laminar airflow ventilation on surgical site infections: a systematic review and meta-analysis. Lancet Infect Dis. February 2017. 
26. Weiser MC, Shemesh S, Chen DD, Bronson MJ, Moucha CS. The Effect of Door Opening on Positive Pressure and Airflow in Operating Rooms. J Am Acad Orthop Surg. 2018;26(5):e105-e113.

27. Birgand G, Toupet G, Rukly S, et al. Air contamination for predicting wound contamination in clean surgery: A large multicenter study. Am J Infect Control. 2015;43(5):516-521. 
Figure 1. Flow chart of procedures included in the analysis and data collected during orthopaedic and cardiac surgery.

Figure 2. Boxplots describing the variability of (A) $\log { }_{10} 0.3 \mu \mathrm{m}$ particle counts $(\mathrm{n}=59$ procedures), (B) the frequency of door openings per hour ( $n=59$ procedures) and (C) cumulated movements by the team per hour $(n=34$ procedures), according to the surgical specialty, the operating rooms and the type of ventilation system.

Table 1. Descriptive analysis on door openings from cutaneous incision to closure during orthopaedic and cardiac surgery.

Table 2. Results of the univariate and multivariate linear mixed models for the particles Log10 $0.3 \mu \mathrm{m}(\mathrm{n}=1747$ samples) and the air microbial count ( $\mathrm{n}=177$ samples) during the 59 included interventions.

Table 3. Results of the univariate and multivariate linear mixed models to evaluate the effect of the number of persons and staff movements on the particles Log10 $0.3 \mu \mathrm{m}$ during the 34 interventions with video data and 1072 particle counts.

\section{SUPPLEMENTARY MATERIAL}

Appendix Table A1. Distribution of particle counts, airborne bacteria and wound contamination at three time points during the 59 procedures with the recording of door openings only.

Appendix Table A2. Evaluation of the Hawthorne effect by the description of door openings during procedures with and without the presence of camera in the operating room.

Appendix Figure A1. Timeline of variable selected for the analysis of the relationship between behaviors and the surrogates of the infectious risk.

Appendix Figure A2. Linear regression of particle counts according to the time, by specialty and by type of ventilation system. 